Lee Tuo-Yeong, Mathematics and Mathematics Education Academic Group, National Institute of Education, Nanyang Technological University, 1 Nanyang Walk, Singapore 637616, Republic of Singapore. e-mail:

tylee@nie.edu.sg

\title{
A SOLUTION TO PFEFFER'S PROBLEM
}

\author{
Abstract \\ We give an example of a non-integrable function $f$ on $[0,1] \times[0,1]$ \\ such that$$
\int_{\alpha}^{\beta}\left(\int_{\gamma}^{\delta} f(x, y) d y\right) d x=\int_{\gamma}^{\delta}\left(\int_{\alpha}^{\beta} f(x, y) d x\right) d y
$$ \\ for each subinterval $[\alpha, \beta] \times[\gamma, \delta]$ of $[0,1] \times[0,1]$. \\ In $[9$, problem 6.2], Pfeffer posed the following problem:
}

Problem. Let $f$ be a function on an interval $A=[a, b] \times[c, d]$, and

$$
\int_{\alpha}^{\beta}\left(\int_{\gamma}^{\delta} f(x, y) d y\right) d x=\int_{\gamma}^{\delta}\left(\int_{\alpha}^{\beta} f(x, y) d x\right) d y
$$

for each subinterval $[\alpha, \beta] \times[\gamma, \delta]$ of $A$. Is $f$ integrable in $A$ ? If so is

$$
\int_{A} f=\int_{a}^{b}\left(\int_{c}^{d} f(x, y) d y\right) d x ?
$$

In the above problem, "integrable" is understood to be in the sense of [8, Definition 3.1]. For other equivalent definitions of this integral, see [4]. By taking $A$ to be the unit square $[0,1] \times[0,1]$, we give a negative answer to the above problem.

Theorem. There exists a non-integrable function $f$ on $[0,1] \times[0,1]$ such that

$$
\int_{\alpha}^{\beta}\left(\int_{\gamma}^{\delta} f(x, y) d y\right) d x=\int_{\gamma}^{\delta}\left(\int_{\alpha}^{\beta} f(x, y) d x\right) d y
$$

for each subinterval $[\alpha, \beta] \times[\gamma, \delta]$ of $[0,1] \times[0,1]$.

Key Words: integrable, integral

Mathematical Reviews subject classification: 26A39

Received by the editors January 26, 2001 
Proof. We shall construct a sequence of subintervals of $[0,1] \times[0,1]$ similar to that of [7, Lemma 4.1]. Then we construct the required function $f$. For each positive integer $n$, we let $a_{n}=\sum_{k=1}^{n}\left(\frac{1}{2}\right)^{k}, c_{n}=\frac{1}{2}\left(a_{n}+a_{n+1}\right)$ and $I_{n}=$ $\left[a_{n}, a_{n+1}\right] \times\left[a_{n}, a_{n+1}\right]$. Define $f_{n}: I_{n} \rightarrow \mathbb{R}$ by

$$
f_{n}(x, y)= \begin{cases}\frac{4}{\left(a_{n+1}-a_{n}\right)^{2}} & \text { if }(x, y) \in\left(\left(a_{n}, c_{n}\right) \times\left(a_{n}, c_{n}\right)\right) \\ \frac{-4}{\left(a_{n+1}-a_{n}\right)^{2}} & \text { if }(x, y) \in\left(\left(c_{n}, a_{n+1}\right) \times\left(c_{n}, a_{n}\right) \times\left(a_{n}, c_{n+1}\right)\right) \\ & \cup\left(\left(c_{n}, a_{n+1}\right) \times\left(c_{n}, a_{n}\right)\right) \\ 0 & \text { otherwise. }\end{cases}
$$

Now define $f:[0,1] \times[0,1] \longrightarrow \mathbb{R}$ by

$$
f(x, y)= \begin{cases}f_{n}(x, y) & \text { if }(x, y) \in I_{n} \text { for some positive integer } n \\ 0 & \text { otherwise. }\end{cases}
$$

By our definition of $f$, we have

$$
\left|\int_{\left[a_{n}, c_{n}\right] \times\left[a_{n}, c_{n}\right]} f\right|=1
$$

for all positive integers $n$. Since the two-dimensional Lebesgue measure of $\left[a_{n}, c_{n}\right] \times\left[a_{n}, c_{n}\right]$ tends to zero as $n \rightarrow \infty$, it follows from [8, Proposition 4.10] and (1) that $f$ is not integrable on $[0,1] \times[0,1]$. However, we shall prove that

$$
\int_{\alpha}^{\beta}\left(\int_{\gamma}^{\delta} f(x, y) d y\right) d x=\int_{\gamma}^{\delta}\left(\int_{\alpha}^{\beta} f(x, y) d x\right) d y
$$

for each subinterval $[\alpha, \beta] \times[\gamma, \delta]$ of $[0,1] \times[0,1]$.

If $[\alpha, \beta] \times[\gamma, \delta] \subset[0,1] \times[0,1]$ with $([\alpha, \beta] \times[\gamma, \delta]) \cap\{(1,1)\}=\emptyset$, then $f$ is Lebesgue integrable on $[\alpha, \beta] \times[\gamma, \delta]$, so (??) is true for all such subintervals of $[0,1] \times[0,1]$. It remains to verify that

$$
\int_{\alpha}^{1}\left(\int_{\gamma}^{1} f(x, y) d y\right) d x=\int_{\gamma}^{1}\left(\int_{\alpha}^{1} f(x, y) d x\right) d y
$$

Let $q$ be the minimum positive integer such that $((\alpha, 1] \times(\gamma, 1]) \cap I_{q} \neq \emptyset$. If $a_{i} \leq x \leq a_{i+1}$ for some $i>q$, then we have

$$
\int_{\gamma}^{1} f(x, y) d y=\int_{a_{i+1}}^{1} f(x, y) d y+\int_{a_{i}}^{a_{i+1}} f(x, y) d y+\int_{\gamma}^{a_{i}} f(x, y) d y=0 .
$$


If $x \in\left[a_{q}, a_{q+1}\right]$, then we have

$$
\int_{\gamma}^{1} f(x, y) d y=\int_{a_{q+1}}^{1} f(x, y) d y+\int_{\gamma}^{a_{q+1}} f(x, y) d y=\int_{\gamma}^{a_{q+1}} f(x, y) d y .
$$

Thus we have

$$
\int_{\gamma}^{1} f(x, y) d y= \begin{cases}\int_{\gamma}^{a_{q+1}} f(x, y) d y & \text { if } x \in\left[a_{q}, a_{q+1}\right] \\ 0 & \text { otherwise. }\end{cases}
$$

Similarly, we have

$$
\int_{\alpha}^{1} f(x, y) d x= \begin{cases}\int_{\alpha}^{a_{q+1}} f(x, y) d x & \text { if } y \in\left[a_{q}, a_{q+1}\right] \\ 0 & \text { otherwise. }\end{cases}
$$

From the above computations, we have

$$
\int_{\alpha}^{1}\left(\int_{\gamma}^{1} f(x, y) d y\right) d x=\int_{\alpha}^{a_{q+1}}\left(\int_{\gamma}^{a_{q+1}} f(x, y) d y\right) d x
$$

and

$$
\int_{\gamma}^{1}\left(\int_{\alpha}^{1} f(x, y) d x\right) d y=\int_{\gamma}^{a_{q+1}}\left(\int_{\alpha}^{a_{q+1}} f(x, y) d x\right) d y .
$$

So (3) is true. Thus $f$ is the function with the desired properties.

Remark The above function $f$ is neither $M_{1}$ (see [2]) nor strongly $\rho$-integrable (see [5]) on $[0,1] \times[0,1]$. In particular, this function cannot be HenstockKurzweil integrable on $[0,1] \times[0,1]$ (see $[6]$ ). If a function is integrable in the sense of [8, Definition 3.1], then it is shown in [4] that it is also $\rho$-integrable (see [3]) there with the same integral value. Must this function be strongly $\rho$-integrable on this interval?

Acknowledgement. The author would like to thank the anonymous referee for posing the following interesting open problems for the BV-integral. For this integral, the reader may wish to consult [1] and references therein.

1. Assuming that the double integral and the iterated integrals exist, do they have the same value?

2. Assuming that the double integral exists and the iterated integrals are equal, is their common value equal to the double integral? 


\section{References}

[1] Z. Buczolich. Thierry De Pauw and W.F. Pfeffer, Charges, BV functions, and Multipliers for Generalized Riemann integrals, Indiana Univ. Math. J., Vol 48(1999), 1471-1511.

[2] J. Jarńik, J. Kurzweil, S. Schwabik, On Mawhin's approach to multiple nonabsolutely convergent integral, C̆asopis pĕst Mat. 108 (1983), 356-380.

[3] J. Jarnik and J. Kurzweil, Perron-type integration on $n$-dimensional intervals and its properties, Czechoslovak Math. J. 45(120) (1995), no. 1, $79-106$.

[4] J. Kurzweil and J. Jarńik, Equivalent definitions of regular generalized Perron integral, Czech. Math. J. 42 (117) (1992), 365-378.

[5] J. Kurzweil and J. Jarńik, Perron-type integration on $n$-dimensional intervals as an extension of integration of stepfunctions by strong equiconvergence, Czech. Math. J. 46 (121) (1996), 1-20.

[6] Lee Peng Yee and Rudolf Vyborny, The integral, An Easy Approach after Kurzweil and Henstock, Australian Mathematical Society Lecture Series 14, Cambridge University Press 2000.

[7] Lee Tuo Yeong, Chew Tuan Seng and Lee Peng Yee, Characterisation of multipliers for the double Henstock integrals, Bull. Austral. Math Soc 54 (1996), 441- 449 .

[8] W.F. Pfeffer, The divergence Theorem, Trans. Amer. Math. Soc. 295 (1986), 665-685.

[9] W.F. Pfeffer, A Riemann type integration and the fundamental Theorem of Calculus, Rend. Circ. Mat. Palermo 36 (1987), 482-506. 